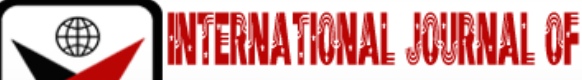

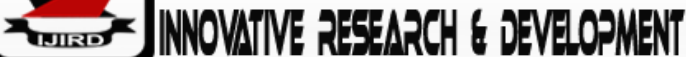

ISSN 2278-0211 (Online)

\section{Enhancing Cell Coverage and Capacity in LTE Heterogenous Network Using Intelligent Base Station}

Ifesinachi E.o.
Engineer, Department of Electronic,
Electronic Development Institute (ELDI), Awka, Nigeria
Idigo V. E.
Professor, Department of Electronic and Computer Engineering,
Nnamdi Azikiwe University Awka, Nigeria
Ohaneme C.o.
Professor, Department of Electronic and Computer Engineering,
Nnamdi Azikiwe University Awka, Nigeria
Obioma P.C.
Engineer, Department of Electronic and Computer Engineering,
Nnamdi Azikiwe University Awka, Nigeria

\section{Abstract:}

The high data traffic demand by mobile users has tremendously increased in recent times, thus creating the need to further improve the capacity and coverage of already existing systems. Recent advancements in self-organizing and self driving Base stations are being exploited to bridge this gap. Although the paradigm have already evolved in $2 G, 3 G$ and $4 G$, the automation is realized by predefined policies, rather than interact with the environment to make smart decisions. In this paper, the eNB's of LTE networks have been designed in such a way that makes them self-aware, selfadaptable and intelligent using the Ant Colony Optimization algorithm. The ACOA was used to minimize the path trailed by the moving eNB's employing the SON capability of LTE systems. Three different scenarios were considered; results showed that in the first scenario, which is considered to be an ideal case, the optimized system improved the system throughput by $19.7 \%$ after 150s. The packet loss for this scenario was also reduced 38\% at 150s. The call blocking probability outperformed the conventional system by $45.9 \%$ as at 120 s and by $22.2 \%$ when the time was 150s. The second scenario considered analyzed the system performance when one cell is overloaded while a nearby cell is idle. Simulation results showed that the ACOA resulted to about $820 \%$ utilization of the idle cell, while each UE achieved an improved throughput compared to the conventional system. Results also showed that the throughput improvement provided by the ACOA in one of the eNB's was about 27Mbps when compared to the conventional system, and $55 \mathrm{Mbps}$ in another $\mathrm{NB}$ when all the cells were loaded to congestion.

Keywords: Het Net, LTE, mobile base station, ant colony, dynamic base station

\section{Introduction}

The fourth generation mobile communication system (4G) network also known as the Long Term Evolution (LTE) cellular network system was developed as an advancement to the former third generation (3G) network that falls within the premise of the third generation project partnership long time evolution 3GPP design by the 3GPP LTE.Due to the high demand by mobile subscribers, high data traffic has tremendously increased in recent times, thereby creating need to search for approaches to further improve the already-known channel capacity and coverage of the already existing systems. For example, in the LTE network systems, the capacity of a network have been improved by (a) improving the spectral efficiency with advanced modulation and coding techniques, (b) improving interference reduction conditions within a cell by using advanced antenna technology (example beam forming), (c) increasing the bandwidth of the radio communication channel, (d) increasing the number of links between a transmitter and a receiver through the use of spatial multiplexing technology (example., MIMO), and (e) adopting aggressive frequency reuse, that is, reusing the same radio resources several times over a given area by deploying more base stations.

The last technique of deploying more base stations has been shown to provide significant network capacity gains since the evolution of cellular networks. Against these improvements, the viability of such improvements may be hampered due to the limitations on the hardware implementation and channel conditions, as well as, the increase in the system complexity due to the use of these advanced techniques. As stated before, in order to increase capacity, cells have to be densely deployed. Beyond the cost implication of these cells, the number of parameters to be configured in these base stations also increases. Also, with the operational complexity in LTE systems, it is challenging and time-consuming for mobile operators to operate and configure the network all by humans. Hence, this is making it necessary to increase 
automation to wireless mobile networks. Currently, many of the autonomous functionalities and automation mechanisms are based on a Self-Organised Network (SON). This is a network concept designed to autonomously configure, optimise and heal LTE HetNets by limiting human intervention [1]. This was first introduced in LTE Release 8 in order to manage mobile cell coverage. This concept relies on artificial intelligence (AI) to analyse the network parameters network and take actions independently [2].Although the self-operation-network (SON) paradigm have already evolved in 2G, 3G and 4G, the automation is realized by predefined policies, rather than interact with the environment to make smart decisions. It is promising to apply AI to the network so as to make it smarter, consequently making the SON coordination to configure, optimize and heal the network more efficiently. Artificial intelligence is the technique that makes machines to solve problems with intelligence like human beings. With artificial intelligence, the machine mimics human minds and 'learns' from the environment, then solve problems by maximizing the success probability. Swarm Intelligence is an AI scheme which is based on observations of collective behaviour in biological organisms like ants and bees, including division of labour, counting larvae, building, cooperative transport, etc. Self-organization protocol via swarm intelligence is a useful solution which has already demonstrated advantages in terms of building an intelligent, self-organizing router which tackles the traditional router problems, as well as balancing the problems of coverage in wireless communications [3]. The Ant Colony Optimisation (ACO) technique is one example of swarm intelligence inspired algorithm that is widely used by researchers to solve problems in LTE systems. This work makes use of the ACO algorithm to make the BTSs self-organized based on pheromone density. The ant agent will be spread throughout the network to find overcrowded and idle or less crowded BTSs and in turn move the dense BTSs loads towards the less dense BTSs.

\section{Review of Related Works}

Many different research models have been proposed to enhance capacity and coverage in LTE with the aim to permit the LTE's attributes to evaluate their needs directly or through the use of equipment such as the coordinate multipoint transmission / reception (CoMP) technique to enhance the equity of traffic in the network. [4] proposed an efficient utilization approach for both lower and higher frequency bands by separating the frequency between wide (macro cells) and local (micro cells) areas. In this work, their approach was used to solve the problem of insufficiency of spectrum in the lower frequency bands. In the study by [5], they used cognitive base stations in LTE networks to enhance the functionality of femtocell base stations. [6] worked on capacity analysis and optimization in heterogeneous network with adaptive cell range control, here the capacity of users in macrocells, small cells and range extensions were analyzed in condition with or without cell range extension (CRE). [7] proposed a scheme that allows mitigation of inter-cell interference through fractional self-powered control performed at each femtocell user. This study analyzes a scheme with optimum power value that provides a compromise between the served uplink signal within unwanted interference plus noise ratio to enhance spectral efficiency in terms of throughput. In the study by [8] on emerging trend on small cell technology, capacity and coverage of indoor environment in a heterogeneous network was enhanced by the deployment of small cell technology like the Metrocell (up to $2 \mathrm{~km})$, microcell $(100 \mathrm{~m}$ to $1000 \mathrm{~m})$, picocell $(50 \mathrm{~m}$ to $100 \mathrm{~m})$ and femtocell $(10 \mathrm{~m}-20 \mathrm{~m})$. This according to the study can doubled the capacity of a HetNet without any addition of spectrum and infrastructure. [9] used particle swarm optimization (POS) algorithm to conduct dynamic user association by finding the optimal bias value via considering the network balance index (the actual load and the predicted load).Minimizing the energy and interferences of mobile users are major concerns of Heterogeneous Networks (HetNet). The work by [10] aimed at improving the energy of mobile users by minimizing a weighted function.In this paper, the ACOA is employed to solve the underlying problem of enhancing cell capacity in LTE systems by considering a densely deployed LTE network in which the BTSs' coverage overlap and the traffic load fluctuate over time and space. The work enhances the system throughput, minimize packet loss, and the load of the system as performance metrics in order to prove the system viability, which most reviewed works failed to ascertain.

\section{The Ant Colony Optimization Algorithm (ACOA)}

The ant colony optimization algorithm (ACO) is an evolutionary meta-heuristic algorithm based on a graph representation that has been applied successfully to solve various hard combinatorial optimization problems. The main idea of ACO is to model the problem as the search for a minimum cost path in a graph. Artificial ants walk through this graph, looking for good paths. Each ant has a rather simple behaviour so that it will typically only find rather poor-quality paths on its own. Better paths are found as the emergent result of the global cooperation among ants in the colony [11].

In 1989 and 1990, in Santa Fe Institute, Bonabeau and his colleagues demonstrated that similar problems involving the best and shortest path can be solved more easily with computers creating 'virtual ants'[11]. According to this, virtual ants leave a scent that represents the length of the route on their behind and by this means the other virtual ants will find the shortest routes. Smell of the traces of long routes that are not preferred will disappear gradually by simulating evaporation of the smell trace at a certain speed. This will prevent the deflection of the virtual ants to the long road outside short cut roads.Figure 1 shows the ants going to their food through a linear way.

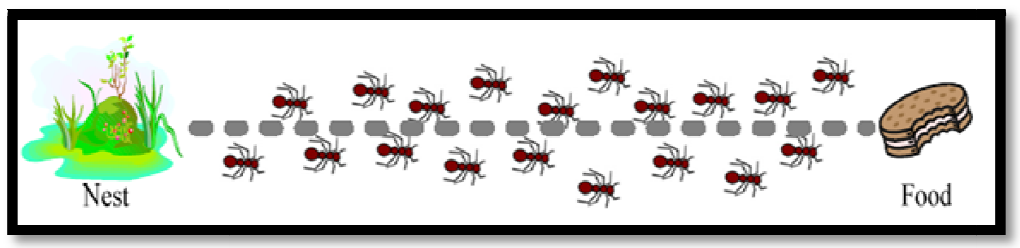

Figure 1: The Path of the Ants to Their Food [11] 


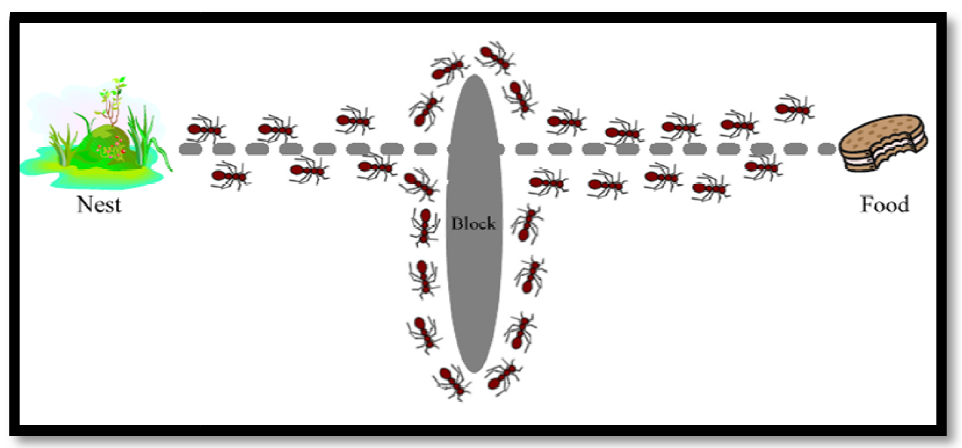

Figure 2: Path Selection by Ants Coming across an Obstacle [11]

Ants help the next ants to choose the path by dropping off stored Pheromones on the chosen way. They show the path of their food using a volatile chemical substance known as pheromone which evaporates with time. A remote ant generally moves randomly and while moving, a quantity of pheromone is deposited by the ant on its trail. Other ants will select the path with the highest pheromone intensity which represents the most attractive way for ants. When an ant follows a path, it strengthens the trail by adding its own pheromone. At the end, this results in a collective behaviour of which there is more than one path with high pheromones; the higher the pheromone, the higher is the number of ants that follow it. This instinctive behaviour explains how they had found the shortest path to their food, even in the case preexisting path cannot be used. In fact, if an obstacle is on the path to food, the ant in front of this obstacle cannot continue and has to make a choice for a new way as shown in figure 2. The basic idea of Ant-based algorithms is that artificial intelligent agents using a simple communication mechanism can produce the solutions to many complex problems. The objective of these algorithms is to enable the network to learn and use that experience for future actions.

\section{Development of the ACO Algorithm}

The ACOA is applied to find the optimal pilot eNB to help it move to dense areas and serve the increased newly arrived UEs. This scheme tackles the problems of fixed coverage scheme, reducing the ratio of coverage holes, proportion of coverage overlaps and probability of blocking new users which enhances the overall performance of the network. Optimization of the new location of the mobile eNB's is modelled by an ant colony movement going from one location to another looking for food. During the food search, the ants will visit the frequently attended cells by the mobile UE. At the end of the algorithm, the pheromone deposited by the ants will accumulate on the cells. As each cell will be served by a mobile eNB, the cell having the most pheromone intensity is considered as the densest cell of the mobile and its serving eNB will require movement to idle or less dense eNB's. This method is also used to predict the moving eNB towards the dense cell of mobile users. The less pheromone quantity cell means less or unused eNB, thus it will be the future moved eNB. The algorithm to predict the movement of the eNBs will be launched when a mobile user enters a location. It creates an N-entries table called Movement Table (MT). The content of this table will be fed by the cache which has history of mobile user's movement behaviour in the case of period (i.e. work day or holiday) or when there is an event (i.e. a football match in a stadium cell). The table will include the most recent entries corresponding to the mobile, with the same source eNB and destination eNB. The number of entries for a mobile user is denoted by N1. If MT is not full which means N1=0, the system will fill it using N2 entries corresponding to other mobiles with the same source cell and the same period or event which assume that mobile users in the same cell can have the same direction.

The algorithm will ensure that eNB's managing the cells can update their users' history movements or directions towards other eNB's and deduce their future locations according to their current location and their behaviours. If a new mobile user enters in the system and the eNB does not have history for it, it can use the history of other mobile users that have the same mobility profile. In the case of public events such as a match in a stadium, a new user will more likely travel from the city, to the stadium.

The system then creates a colony of ants whose size is equal to the movement table (MT) size. Each ant $a_{i}, \forall i=$ $1, \ldots . N$ is associated with the line i of the movement table. Two fields are used to define the structure of an ant, the visibility field and the pheromone field. The visibility field is considered as a vectorn of R elements corresponding to the number of adjacent cells. Each element of this vector represents the visibility of an adjacent cell by an ant. This field is activated to reflect the fact that ants prefer an already visited cell when they search for food. If $\eta_{i, k}$ represents the visibility of the cell for ant $a_{i}$ then:

$\eta_{a_{i}, k}=\left\{\begin{array}{c}X i f M T_{i}(\text { DestinationeNB }) \neq{\text { korif } M T_{i} \text { isnull }} \\ X . \mu \text { if } M T_{i}(\text { Destinatione } B)=k\end{array}\right.$

Where $k=1, \ldots R$ which represents the adjacent cells, $X$ is a value $>0$ and $\mu>1$ is the parameter to increase the cell degree of visibility if it was already visited by the mobile. $M T_{i}$ represents the $i^{\text {th }}$ entry of the movement table. To keep the trace of the pheromone deposited by the ants, we use a vector $\tau$ with $\mathrm{R}$ elements.

Each element of the vector corresponds to the quantity of pheromone in a corresponding cell. This vector will be initialized to $\tau_{k}\left(t_{0}\right)=Q$ for $k=1, \ldots R$ (where $\mathrm{k}$ represents the cell $\mathrm{k}$ and $t_{0}$ represents the initial time).

The search process proceeds in a set of iterations in which each ant moves towards an adjacent cell, deposits the quantity $\mathrm{Q}$ of pheromone in this cell to encourage the other ants to go towards it. It then returns towards its nest (i.e., the 
current cell) in order to repeat the same process. Each ant chooses the future cell according to its degree of visibility and the intensity in pheromone of the cell. At any moment t, the ants $a_{i}$ chooses to go to the cell K according to the probability:

$P_{a_{i}, j}(t)=\frac{\left[\tau_{i j}(t)\right]^{\alpha}\left[\eta_{a_{i}, k}\right]^{\beta}}{\sum_{j=1}^{R}\left[\tau_{i j}(t)\right]^{\alpha}\left[\eta_{a_{i}, j}\right]^{\beta}}$

After each iteration time $(t+1)$, the pheromone intensity in the locations is updated according to ants' contribution and the evaporation rate. For each location $k$, we have:

$\left[\tau_{k}(t+1)\right]=(1-\rho) \cdot \tau_{k}(t)+n \cdot Q$

Note that a small value of $\rho$ generates slow pheromone dissipation and a high value generates a faster dissipation. Notice that $n$ represents the number of ants that choose location $k$. The proposed algorithm in this work provides the ability to integrate a mobile's behaviour, the existing infrastructure and other mobiles' behaviour in the prediction process (mobility prediction based on an ant system).The flowchart of the system is as shown in figure 3 . From the flow chart, the information obtained by the ACOA from each visited site is sent as a script to the Network Interface Collector (NIC) of the eNB, which will first sense the network state information to decide whether (or not) to move the desired eNB based on the traffic load information, cell density, channel conditions and UE requirements information available.

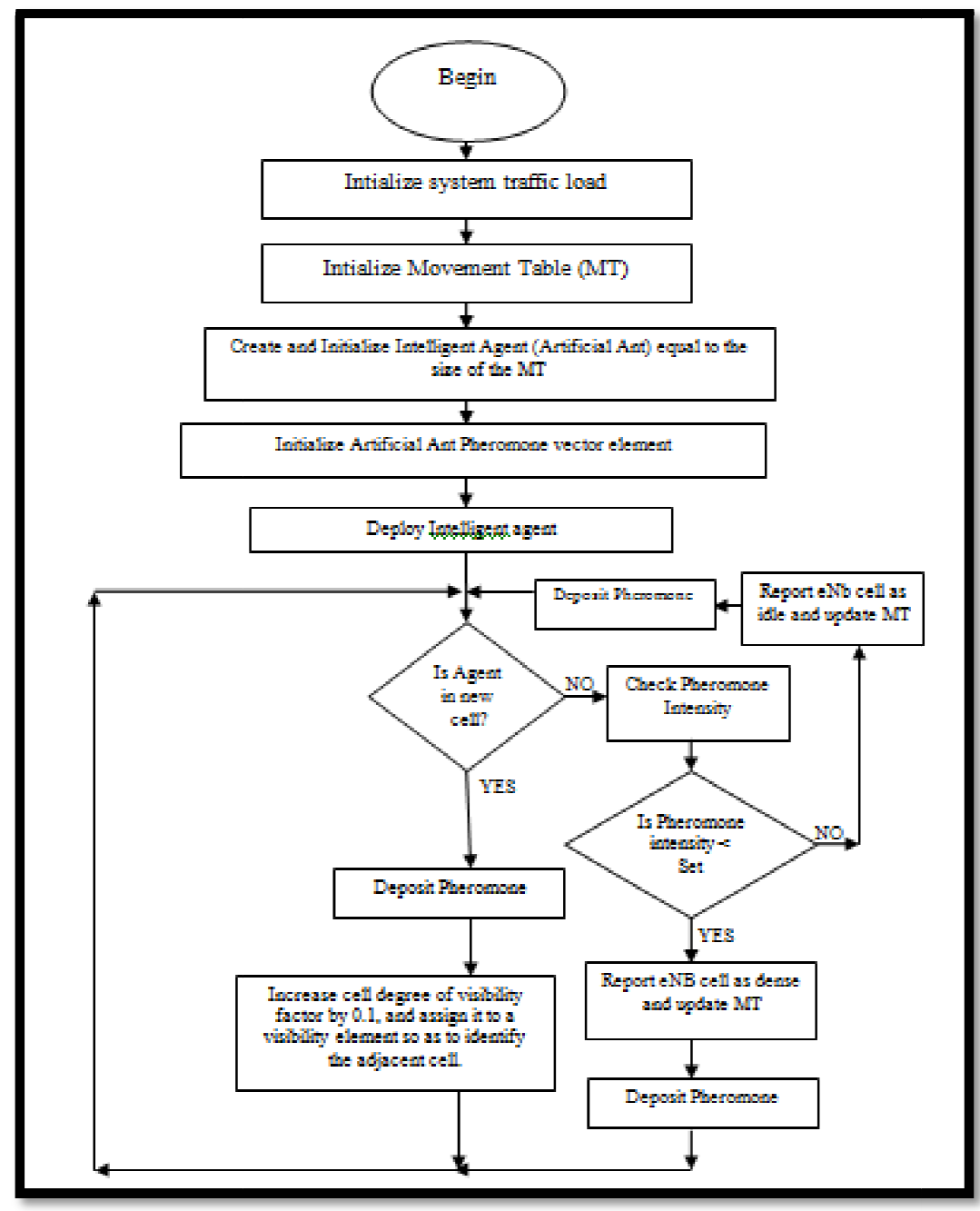

Figure 3: The Flowchart of the System

\section{Simulation Results}

The simulation was conducted using the OPNET simulator. The downlink scenario of a two-tier OFDMA network is considered, and comprises of macrocells and small cells where the former is overlaid with the latter. In each macrocell, an eNB is located at the center of hexagonal coverage area to afford a basic network coverage. Macro users are randomly distributed in the whole area as shown. A network of unit area of $1000 \mathrm{~m} \times 1000 \mathrm{~m}$ was simulated; one node acts as a server and is connected to 3 eNb's with wired links as depicted in figure 4. 


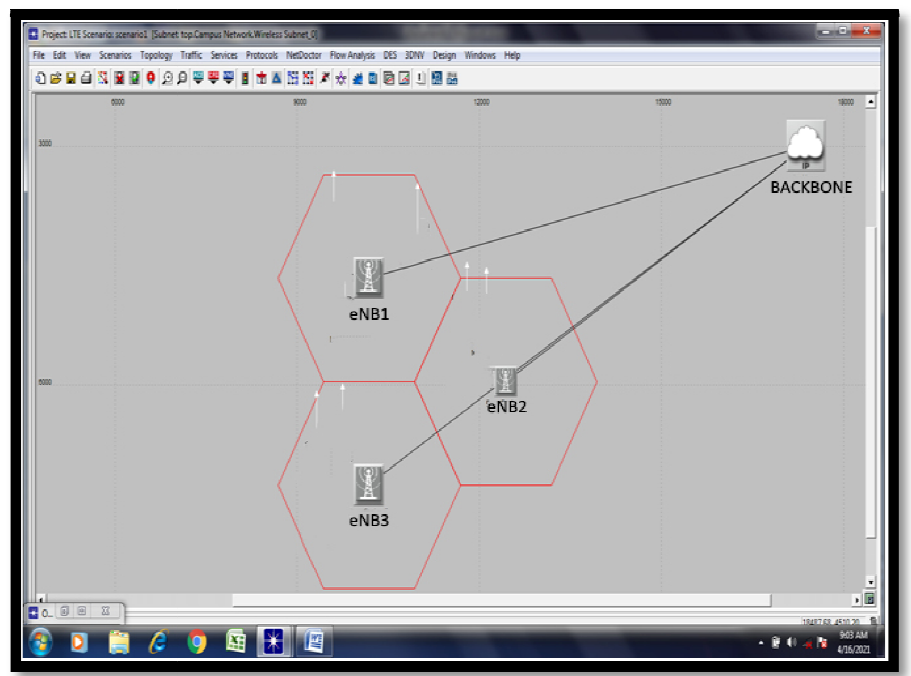

Figure 4: Simulation Environment

The traffic between the eNB's and mobile nodes transmits packets with a Constant Bit Rate (CBR). The packet size is 512 bytes and the simulation time is 150 s. In table 4 , the network parameters are shown.

\begin{tabular}{|c|c|}
\hline Parameter & Value \\
\hline Nodes & 8 \\
\hline Speed & $10 \mathrm{~m} / \mathrm{s}$ \\
\hline Movement & Random \\
\hline MAC & 802.11 \\
\hline Number of Active users & 9 \\
\hline Application & CBR \\
\hline Simulation time & $150 \mathrm{~s}$ \\
\hline Area & $1000 \mathrm{X} 1000 \mathrm{~m}^{2}$ \\
\hline eNodeB coverage area & $100 \mathrm{~m}$ \\
\hline Routing Protocol & DSDV \\
\hline Transmitting capacity & 2 Kbps, $4 \mathrm{Kbps}$ \\
\hline Number of ant agent & 4 \\
\hline Packet Size & 512 bytes \\
\hline Table 1 Shows the Parameters Used In Configuring the
\end{tabular}

Table 1: Shows the Parameters Used In Configuring the Network for This Experiment

The results and analysis of the simulation are presented for the comparison of threedifferent scenarios. The system performance is analysedfor the conventional system and for an optimized system with the use of the ACO algorithm. Figure 5 illustrates an ideal scenario where there is a normal load distribution among the network, and the UE are considered to be mobile.

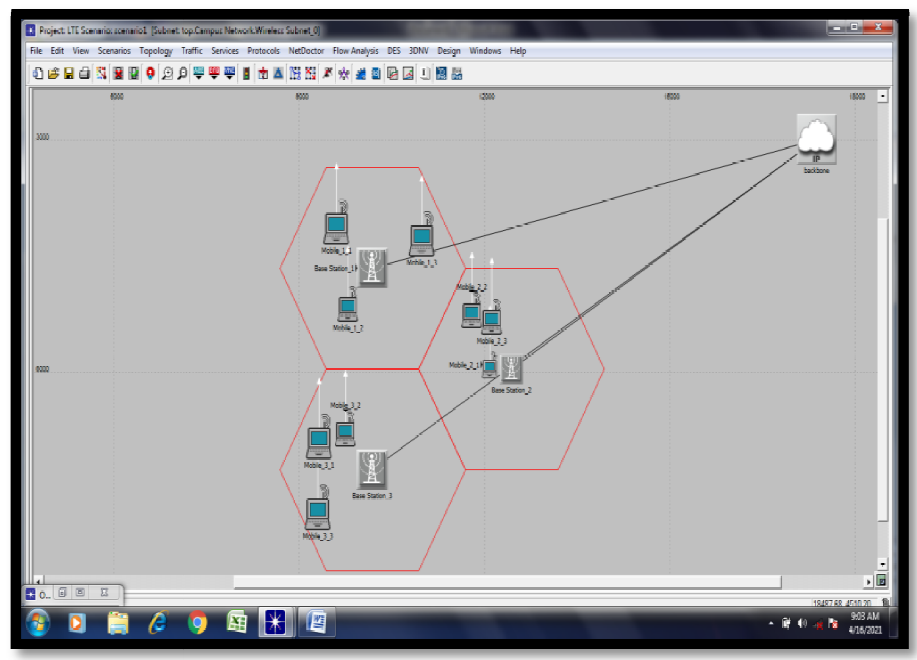

Figure 5: Simulation Scenario 1 


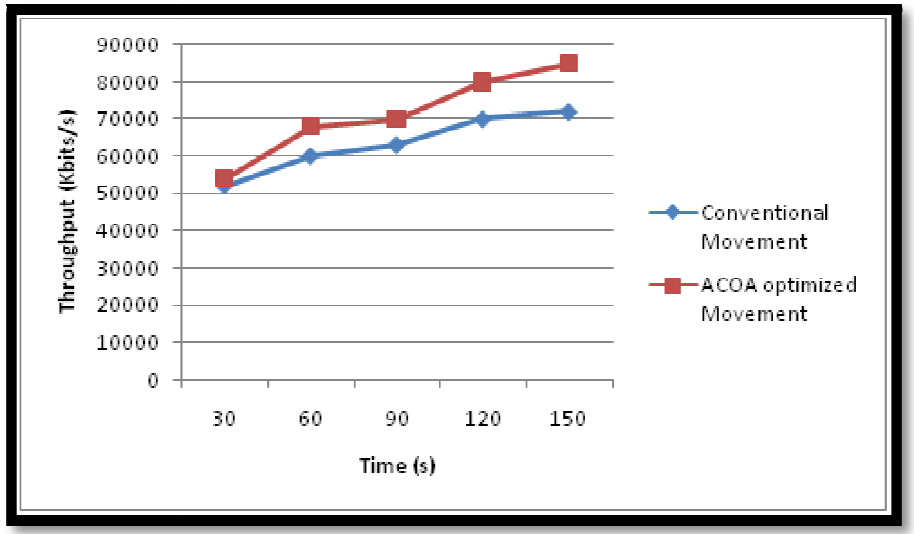

Figure 6: Throughput Network Performance for Conventional Movement and ACOA Optimized Movement for Scenario 1

The scenario represented in figure 5 is a common one in most real life situations, the simulation result in terms of throughput and packet loss is as illustrated in figure 6 and 7.

From the results, it can be found that the scenario where the ACOA algorithm was used, the system significantly outperformed the conventional method. Within the first 30 to 50 seconds, the throughput from both the normal and the optimized algorithm performed fairly similarly, with the optimized algorithm outperforming the normal technique. It can be seen that with time, the performance of the ACOA optimized system increased and became significant from $60 \mathrm{~s}$ seconds with up to $70 \mathrm{Mbps}$ throughput. At 150 seconds, the throughput of the ACO algorithm improved by $19.7 \%$ when compared to the conventional system. The packets lost by both systems were also accessed, so as to determine how well the algorithm performs. The result is as shown in figure 7.

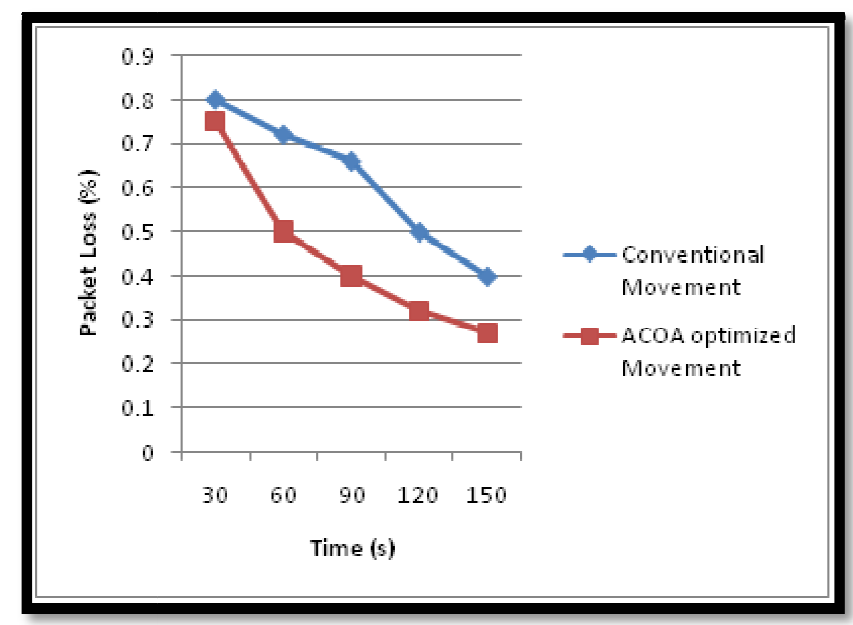

Figure 7: Packet Loss Ratio in the Network for Both Systems for Scenario 1

From the simulation result, the application of the ACOA shows a better reduction in the percentage of packet loss as shown in figure 4.4 for all the times considered. Between 15 to 20s, both system performance was fairly similar, but as the time increased, the ACOA showed better results (i.e., gave shorter paths) to the UE dense locations. Comparing the conventional movement and the ACOA optimized movement, the optimized movement clearly achieved better performance by approximately $39 \%$ at 150 seconds than the normal moving technique. The simulation also considered the user equipment experience, and the result is presented in figure 8. 


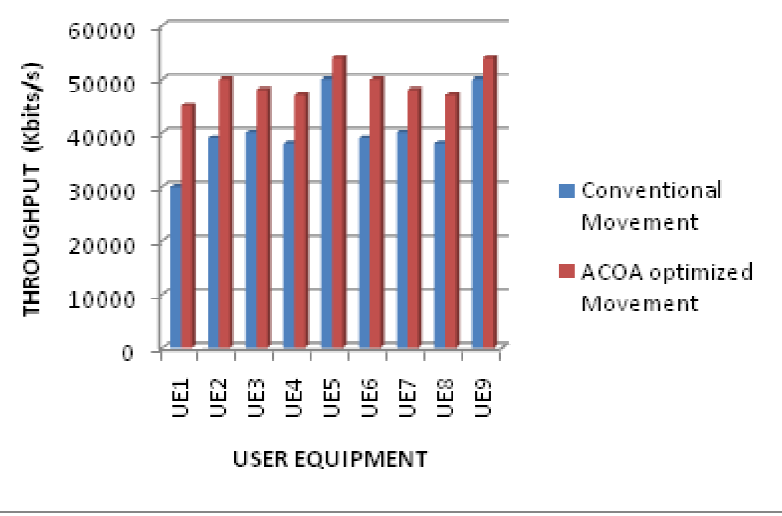

Figure 8: Throughput Network Performance of UE for Both Systems in Scenario 1

From figure 8, it is obvious that in the optimized design, each UE achieved an improved throughput compared to the conventional system. For UE1, the optimized algorithm achieved 14Mbps better than the normal algorithm. Similarly for UE5 where both systems had an improved performance due to user mobility and position in the cell, the optimized algorithm achieved 3Mbits/s better than the conventional system. The ACO algorithm was also applied to the UE2, UE3 and UE4 that are sparsely distributed in the cell; the system employing the ACOA achieved $11 \mathrm{Mbps}$, 8Mbps and 10Mbps respectively better than the conventional system. The call blocking probability is also considered as shown in figure 9 .

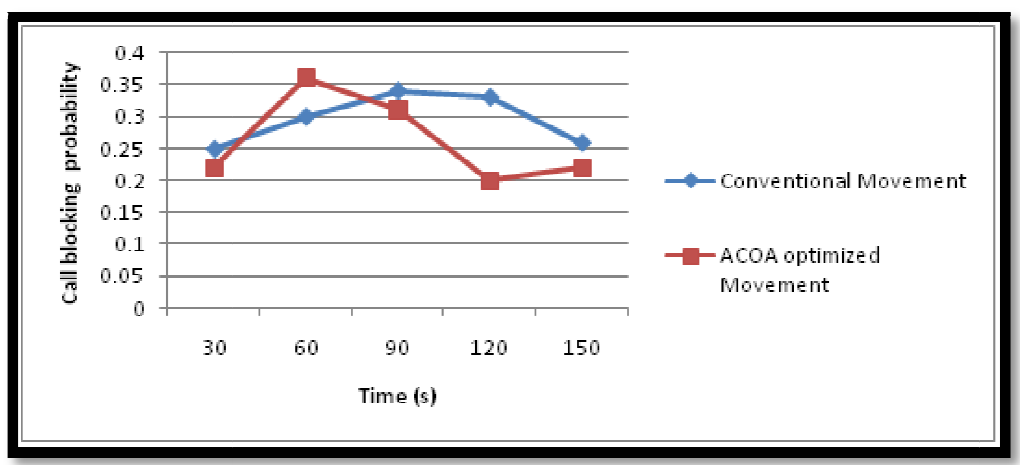

Figure 9: Call Blocking Probability for Scenario 1

As can be seen in figure 9, within the first 30s, the call blocking probability for both systems were fairly close, about 0.22 and 0.25 for the optimized system and conventional system respectively. As the time increased, with UE movement and activity within the cell, the call blocking probability remained fairly low. For the system employing the ACOA, the call blocking probability outperformed the conventional system by $45.9 \%$ as at 120 s and by $22.2 \%$ when the time was 150s.These investigations were further extended to throughputs achieved at each eNBs when there's a major event happening close to a particular eNB site.

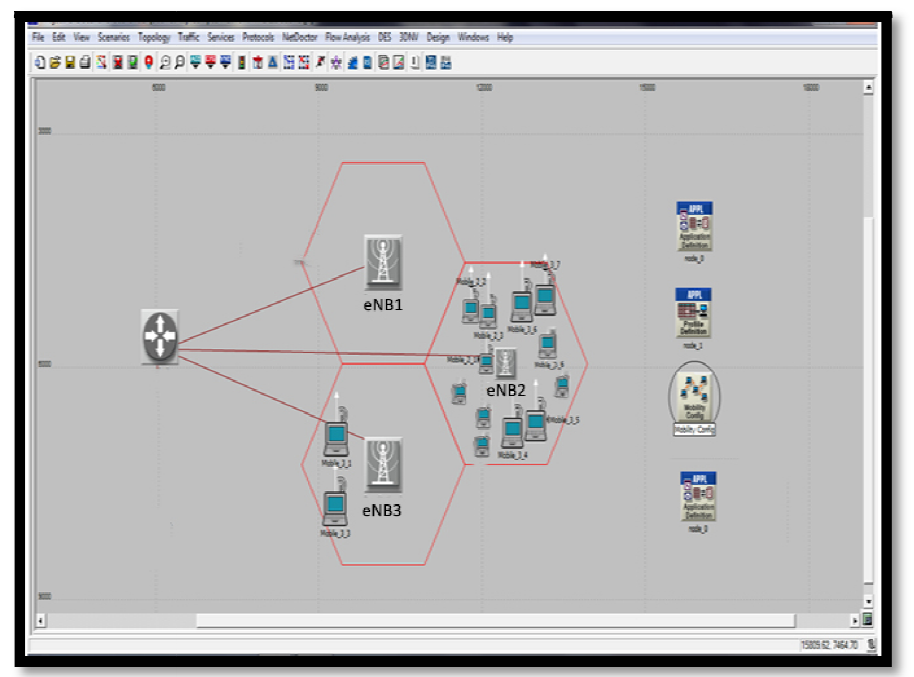

Figure 10: Simulation Scenarios 2

When the density of the cells was increased as shown in figure 10, the system performance at each of the eNB's was monitored. From figure 10, the system at eNB2 was loaded to congestion as shown and some UE sparsely distributed 
at eNB3. This scenario is likened to when there is an event, say a football match and there are clusters of people very close to a particular eNB (in this case eNB2), and a close by cell is idle. The result of the performance of the two systems is presented in figure 11 .

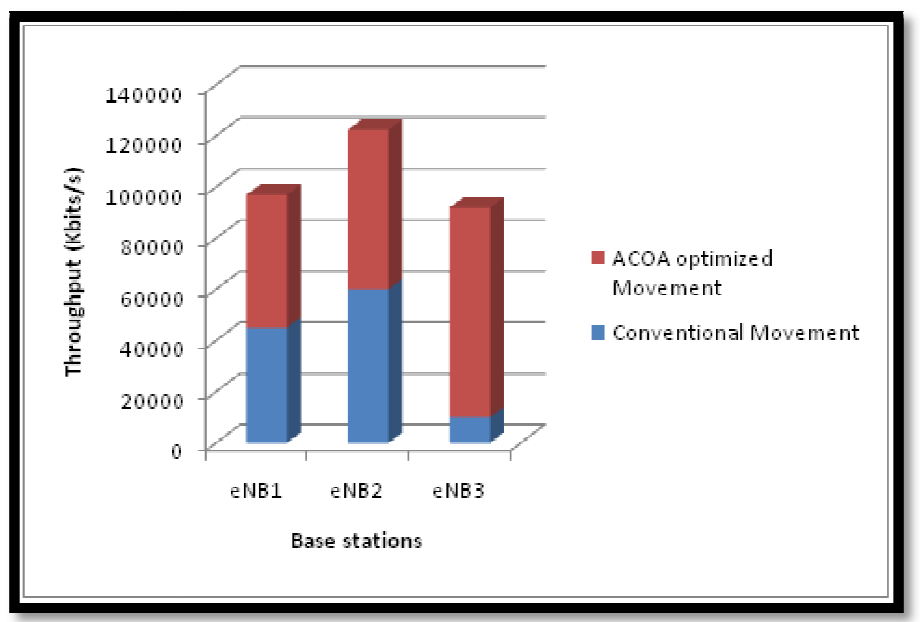

Figure 11: Comparison of Throughput Data When the Enbs Are Moved For Both Systems

From figure 11, the results obtained showed that the ACOA helped to utilize the idle base at eNB1, thus reducing the load at eNB2. The throughput improvement provided by the ACOA in eNB2 even with the density of the cell is about $2.5 \mathrm{Mbps}$ when compared to the conventional system, and 7Mbps and 72Mbps respectively for eNB1 and eNB3. This results to $820 \%$ utilization of an idle cell. Beyond improving the throughput, an added advantage of the optimized system is that the call blocking probability is greatly reduced as shown in figure 12

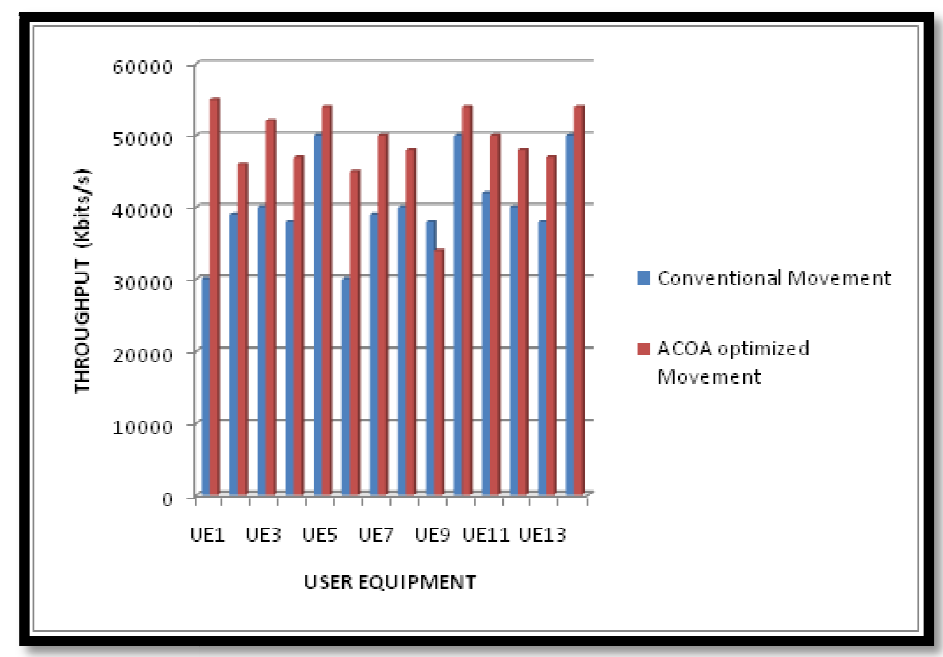

Figure 12: Throughput Network Performance of UE for Both Systems for Scenario 2

From figure 12, it is obvious that in the optimized design, each UE achieved an improved throughput compared to the conventional system. For UE1, the optimized algorithm achieved $25 \mathrm{Mbps}$ better than the normal algorithm. Similarly for UE5 where both systems had an improved performance due to user mobility and position in the cell, the optimized algorithm achieved 4Mbits/s better than the conventional system. The ACO algorithm was also applied to the UE2, UE3 and UE4 that are sparsely distributed in the cell; the system employing the ACOA achieved $7 \mathrm{Mbps}, 12 \mathrm{Mbps}$ and $9 \mathrm{Mbps}$ respectively better than the conventional system. The call blocking probability is also considered as shown in figure 13 . 


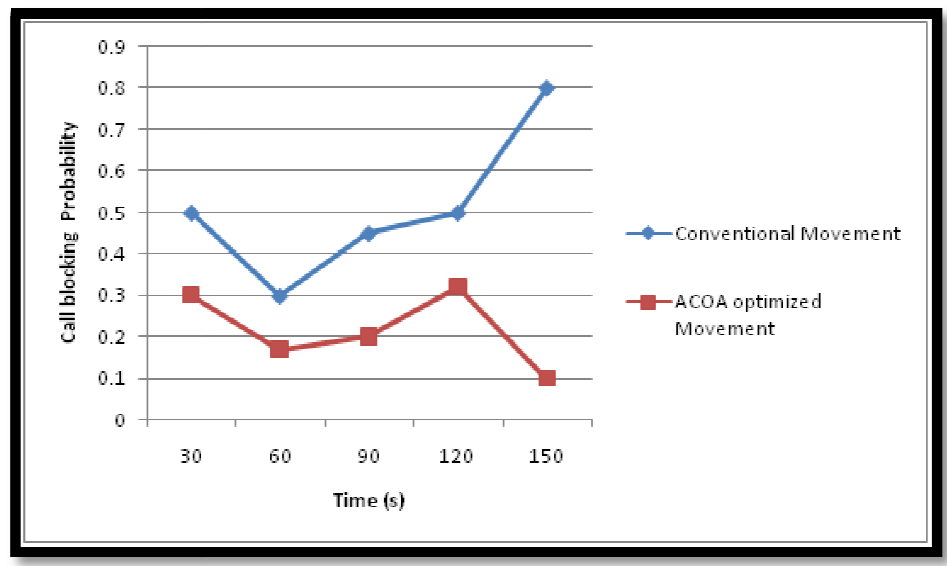

Figure 13: Call Blocking Probability

As can be seen in figure 13, within the first 30s, the call blocking probability for both systems was fairly high, about 0.3 and 0.5 for the optimized system and conventional system respectively. This covers the early periods were there are still little influx of persons migrating into the cell. As the time increased up 150s, the cell is heavily loaded and at this point, the call blocking probability for eNB2 spiked to 0.8 , which meant that there was a high chance for calls being put through in the venue will be blocked. For the system employing the ACOA, the call blocking probability dropped to about 0.1 , making it very difficult for calls to be blocked.

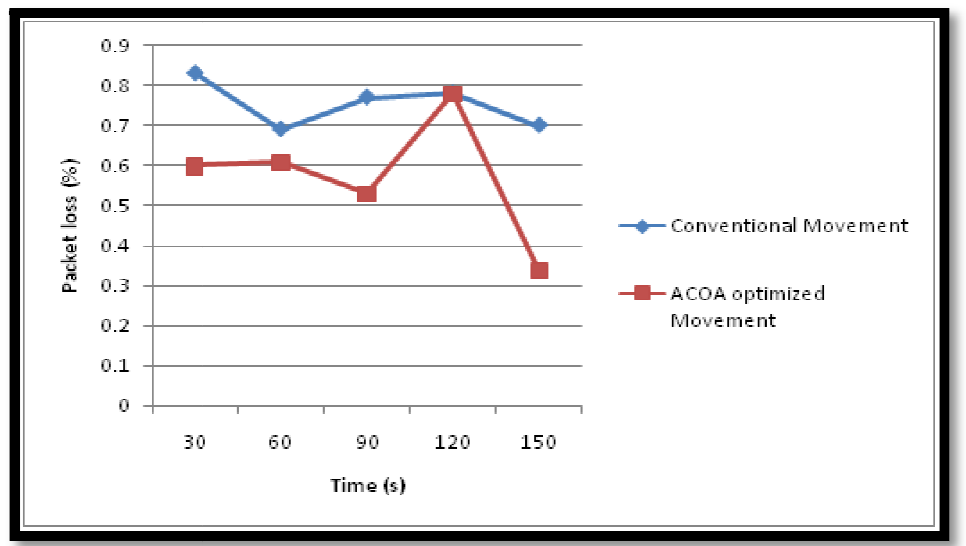

Figure 14: Packet Loss Ratio in the Network for Both Systems for Scenario 3

From the simulation result, the application of the ACOA shows a better reduction in the percentage of packet loss as shown in figure 14 for all the times considered. As at 30s into the simulation, the optimized system already showed $27.7 \%$ reduction in packet loss when compared to the conventional system. From figure 15 its obvious that as the time increased, the ACOA showed better results (i.e., gave shorter paths) to the UE dense locations accept at the 120s. Comparing the conventional movement and the ACOA optimized movement, the optimized movement clearly achieved better performance especially at the 150 s where the packet loss reduction was about $51.4 \%$.A third scenario is also considered where all the cells are congested as shown in figure 15.

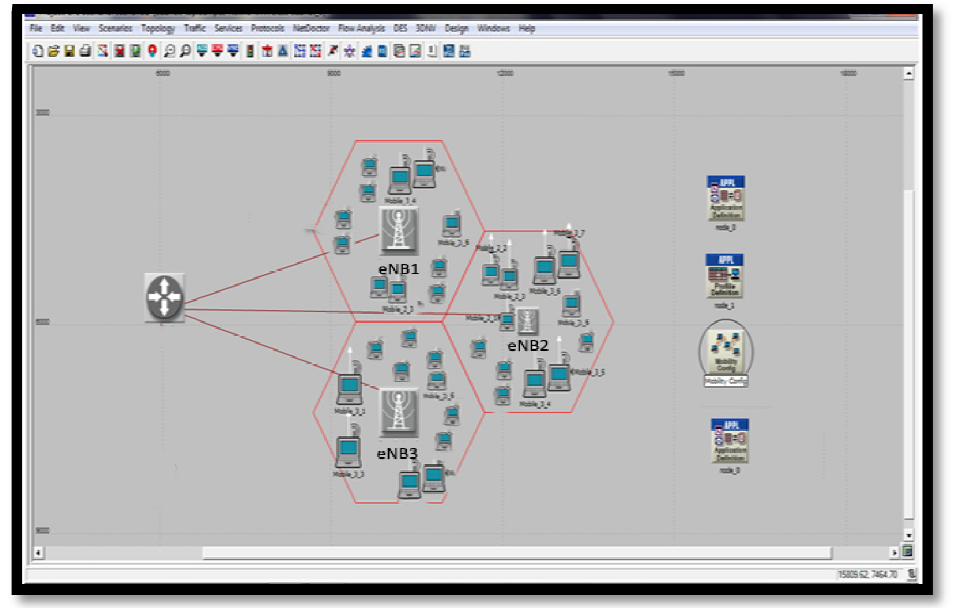

Figure 15: Simulation Scenario 3 
When the density of the cells was increased as shown in figure 16, the system performance at each of the eNB's was monitored. This scenario is likened to when there is a rally and everyone is clustered all over the sites.

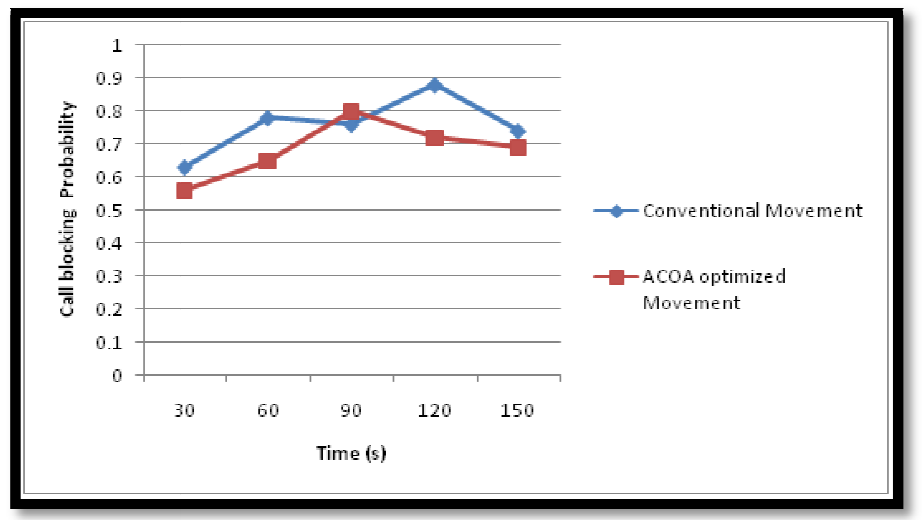

Figure 16: Call Blocking Probability for Scenario 3

As can be seen in figure 16, the call blocking probability for both systems is fairly high given traffic and over loaded nature of the cells. With the optimized system there is a slight reduction in the call blocking probability. As at 30s, the optimized system showed a $11.11 \%$ reduction in the call blocking probability. Though at 90 s, the conventional system performed better than the optimized system by $5 \%$, it can be seem that as the simulation progressed, the optimized system outperformed the conventional system by $6.7 \%$. The performance at the various eNB's were also analyzed and shown in figure 18.

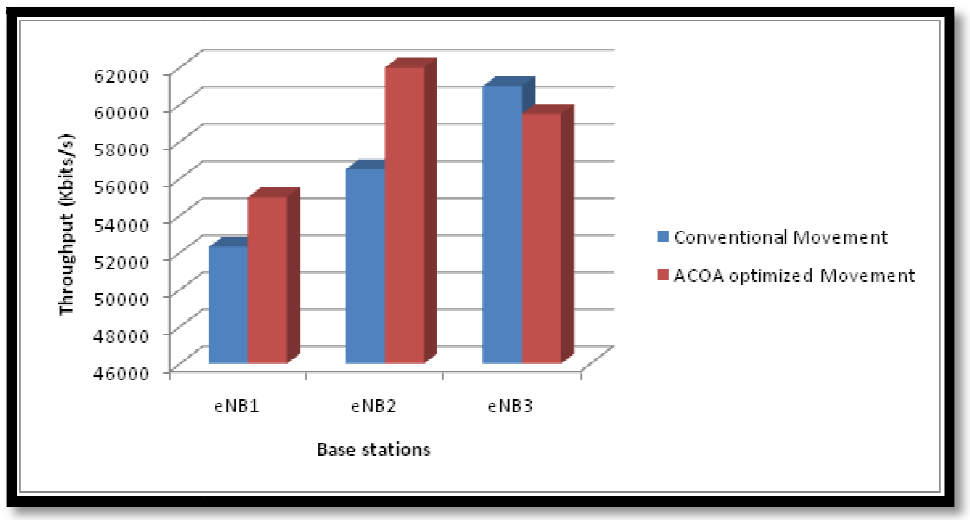

Figure 17: Base Station Performances for Scenario 3

From figure 17, the results obtained showed that the ACOA helped to utilize the base stations at full capacity. The throughput improvement provided by the ACOA in eNB1 even with the density of the cell is about 27Mbps when compared to the conventional system, and 55Mbps for eNB2 (which is about 9.7\% increase). Beyond improving the throughput, an added advantage of the optimized system is that the packet loss is also reduced as shown in figure 19.

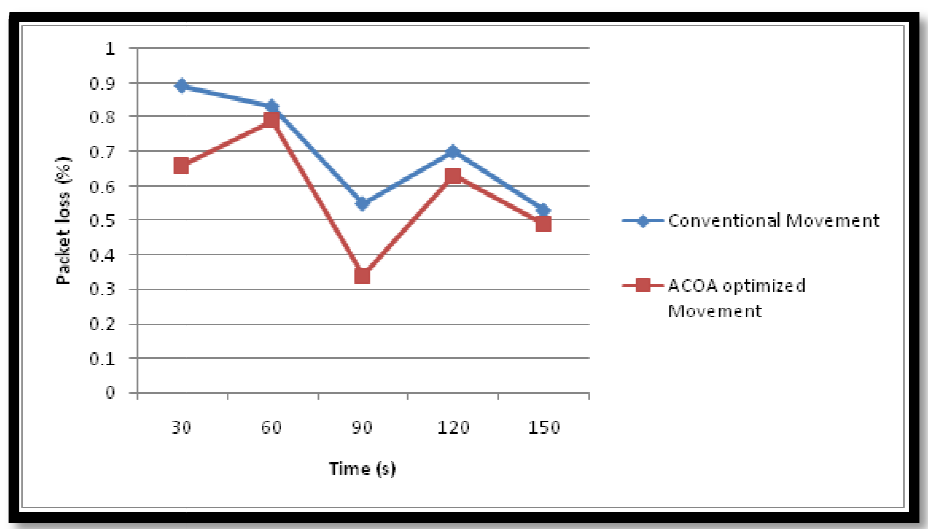

Figure 18: Packet Loss Percentages for Scenario 3

From the simulation result, the application of the ACOA shows a better reduction in the percentage of packet loss as shown in Figure 18for all the times considered. Comparing the conventional movement and the ACOA optimized movement, the optimized movement clearly achieved better performance by approximately $38.2 \%$ at 90 seconds than the normal moving technique. 


\section{Conclusion}

In this paper, the eNb's of LTE networks have been designed in such a way that makes them able to be self-aware, self-adaptable and intelligent using the Ant Colony Optimization algorithm. This algorithm was inspired from the biological ant behaviour when in search for food. The ACOA was used to minimize the path trailed by the moving eNb's employing the SON capability of LTE systems. This technique was deployed to increase the coverage capacity of the system by increasing the system throughput and reducing the packet loss. Three different scenarios were considered, results showed that in the first scenario, which is considered to be an ideal case, the optimized system improved the system throughput by $19.7 \%$ after 150 s. The packet loss for this scenario was also reduced $38 \%$ at 150 s, and by applying the antcolony optimization algorithm to the moving BTSs technique, improved the throughput significantly by up to 40 Mbps and reduced packet loss rate in the network by up to 19\%. For the system employing the ACOA, the call blocking probability outperformed the conventional system by $45.9 \%$ as at 120 s and by $22.2 \%$ when the time was 150 s.

In the second scenario, the system at eNB2 was loaded to congestion and some UE sparsely distributed at eNB3. This scenario is likened to when there is an event, say a football match and there are clusters of people very close to a particular eNB (in this case eNB2), and a close by cell is idle, the results obtained showed that the ACOA helped to utilize the idle base at eNB1, thus reducing the load at eNB2. The throughput improvement provided by the ACOA in eNB2 even with the density of the cell is about $2.5 \mathrm{Mbps}$ when compared to the conventional system, and $7 \mathrm{Mbps}$ and $72 \mathrm{Mbps}$ respectively for eNB1 and eNB3. This result to 820\% utilization of an idle cell, while each UE achieved an improved throughput compared to the conventional system. For UE1, the optimized algorithm achieved 25Mbps better than the normal algorithm. For the system employing the ACOA, the call blocking probability dropped to about 0.1 , making it very difficult for calls to be blocked. A third scenario is also considered where all the cells are congested. When the density of the cells was increased, the system performance at each of the eNB's was monitored. This scenario is likened to when there is a rally and everyone is clustered all over the sites. The call blocking probability for both systems was fairly high, with the optimized system there was a slight reduction in the call blocking probability. As at 30s, the optimized system showed a 11.11\% reduction in the call blocking probability. The throughput improvement provided by the ACOA in eNB1 even with the density of the cell was about 27Mbps when compared to the conventional system, and 55Mbps for eNB2 (which is about $9.7 \%$ increase).

NS3 is an alternative network simulation tool that could be used in the study of network architectures, technologies and topologies. This software is worth trying out since it provides results comparable to that of OPNET. More scenarios with sophisticated configuration examples under different density settings are suggested to be considered for the future study of this work. With regards to the bio-inspired optimization techniques to move the appropriate BTS and balance the number of mobile users allocated to each BTS, further study about other bio-inspired techniques to build the SON capabilities is recommended.

\section{References}

i. D. Lopez-Perez, I. Guvenc, G. De la Roche, M. Kountouris, T. Q. Quek, and J. Zhang, 'Enhanced intercell interference coordination challenges in heterogeneous networks,' IEEE Wireless Communications, vol. 18, pp. 22-30, 2011

ii. Sesia. S, Toufik. I, and Baker .M, LTE - The UMTS Long Term Evolution: From Theory toPractice, John Wiley \& Sons, Ltd, Chichester, UK, 2012.

iii. K Son, H Kim, Y Yi, B Krishna machari, 'Base station operation and user Association mechanisms for energy-delay tradeoffs in green cellular Networks'. Selected Areas Commun. IEEE J. 29(8), 1525-1536, 2011Attar, V. Krishnamurthy, and 0. N. Gharehshiran, 'Interference management using cognitive base-stations for UMTS LTE,'IEEE Communications Magazine, vol. 49, pp. 152-159, 2011

iv. Yen-Wei Kuo and Li-Der Chou, 'Fuzzy-based coverage and capacity scheme in LTE heterogeneous networks' Journal of the Chinese Institute of Engineers, https://doi.org/10.1080/02533839.2017.1384323, 2017

v. RebenKurda, 'Heterogeneous networks: Fair power allocation in LTE-A uplink scenarios'https://doi.org/10.1371/journal.pone.0252421, Accessed 16th June 2021

vi. Andrea Tassi, ChadiKhirallah, DejanVukobratovic, Francesco Chiti, John S. Thompson and Romano Fantacci, 'Resource Allocation Strategies for NetworkCodedVideo Broadcasting Services over LTE-Advanced' IEEE Transactions onVehicular Technology, pages 1-12, 2014

vii. M. R. Tabany and C. G. Guy, 'Design and implement delay-aware QoS scheme for 3GPP LTE/LTE-A networks for mixed traffic flow,' IEEE Symposium on Computers and Communication (ISCC), pp.38-44, 2015

viii. Samira Achki, Layla Aziz, Fatima Gharnati, Abdellah Ait Ouahman, 'User Association Strategy for Energy Efficiency and Interference Mitigation of Heterogeneous Networks', Advances in Materials Science and Engineering, vol. 2, 2020, Article ID 7018727, https://doi.org/10.1155/2020/7018727

ix. M. Ünal et al'Optimization of PID Controllers Using AC and GA', SCI 449, pp. 31-35., 2012 